\title{
A proteogenomic analysis of Shigella flexneri using 2D LC-MALDI TOF/TOF
}

Lina Zhao ${ }^{1,2}$, Liguo Liu' ${ }^{1}$ Wenchuan Leng ${ }^{1}$, Candong Wei ${ }^{1^{*}}$ and Qi Jin $^{1 *}$

\begin{abstract}
Background: New strategies for high-throughput sequencing are constantly appearing, leading to a great increase in the number of completely sequenced genomes. Unfortunately, computational genome annotation is out of step with this progress. Thus, the accurate annotation of these genomes has become a bottleneck of knowledge acquisition.

Results: We exploited a proteogenomic approach to improve conventional genome annotation by integrating proteomic data with genomic information. Using Shigella flexneri 2 a as a model, we identified total 823 proteins, including 187 hypothetical proteins. Among them, three annotated ORFs were extended upstream through comprehensive analysis against an in-house N-terminal extension database. Two genes, which could not be translated to their full length because of stop codon 'mutations' induced by genome sequencing errors, were revised and annotated as fully functional genes. Above all, seven new ORFs were discovered, which were not predicted in S. flexneri 2a str.301 by any other annotation approaches. The transcripts of four novel ORFs were confirmed by RT-PCR assay. Additionally, most of these novel ORFs were overlapping genes, some even nested within the coding region of other known genes.

Conclusions: Our findings demonstrate that current Shigella genome annotation methods are not perfect and need to be improved. Apart from the validation of predicted genes at the protein level, the additional features of proteogenomic tools include revision of annotation errors and discovery of novel ORFs. The complementary dataset could provide more targets for those interested in Shigella to perform functional studies.
\end{abstract}

\section{Background}

New sequencing strategies are constantly under development and are currently able to process a large number of samples with great efficiency in a short period of time. However, accurate annotation of the resulting sequenced genomes has become the bottleneck of knowledge acquisition. Conventionally, most genome sequences are annotated with multiple gene prediction algorithms such as GLIMMER, CRITICA, and GeneMark, or by manual assignment based on BLAST search results $[1,2]$. Gene density is sufficiently high in prokaryotes, such that coding sequences (CDSs) frequently overlap. Moreover, exonintron structures present in eukaryotic genomes also make computational annotation difficult. These annotations are

\footnotetext{
* Correspondence: weicando@ipbcams.ac.cn; zdsys@vip.sina.com 'State Key Laboratory for Molecular Virology and Genetic Engineering, Institute of Pathogen Biology, Chinese Academy of Medical Sciences \& Peking Union Medical College, Beijing, PR China

Full list of author information is available at the end of the article
}

rarely experimentally validated, though in silico annotation methods could be executed with both high speed and good coverage [3]. The predicted genes exhibit frequent errors, particularly in false recognition of alternative start codons, underestimate of short CDSs, misannotation of pseudogenes, and confusion over overlapping genes. Previous studies have demonstrated that error rates in the definition of translation start sites (TSSs) varied from 10\% to $40 \%$ in some bacterial and archaeal genomes, according to different computational methods used $[4,5]$. Likewise, after analysis of overlaps larger than $60 \mathrm{bp}$ among 338 prokaryotic genomes, it was found that the annotation of most previously identified genes was incorrect [6]. In these cases, computational methods were unable to recognize mutations induced by sequencing errors, such as frameshifts and stop codon mutations. As such, there is a great need for further experimental validation or complementary annotation approaches for conventional genome annotation.
Ciomed Central

(c) 2011 Zhao et al; licensee BioMed Central Ltd. This is an Open Access article distributed under the terms of the Creative Commons Attribution License (http://creativecommons.org/licenses/by/2.0), which permits unrestricted use, distribution, and reproduction in any medium, provided the original work is properly cited. 
Currently, mass spectrometry (MS)-based proteomic methods are used to address difficulties in gene annotation. Unambiguous identification of proteins by MS is more explicit and confident than that from genomic sequence data alone. The high-throughput nature of shotgun proteomics makes this technology cost-effective and readily reliable to the automated genome annotation process $[7,8]$. Integrating proteomic information into the genome annotation process, termed proteogenomics [9], directly maps tandem mass spectrometry (MS/MS) spectra data against all six possible reading frames from raw genomic DNA sequences, i.e., experimental proteomic data can be fed back to the genome to aid in the validation of predicted protein-coding genes, potentially avoiding any biases in the computer algorithm. Proteogenomics analysis have already been applied to a number of sequenced prokaryotes and eukaryotes such as the Mycoplasma pneumonia [7], Mycobacterium lepra [10], Shewanella oneldensis [11], Mycoplasma mobile [12], Toxplasma gondii [13], Arabidopsis thaliana [14], human [15] and so on. As a complementary annotation approach, proteomic methods are important for improving the quality of genome annotation, especially for correction of start codon errors by the analysis of a new framework and sequencing of $\mathrm{N}$ terminally acetylated peptides [16-18] and discovery of novel genes missed in the computational genome annotation process [19-23]. Although proteogenomics has made great progress in recent past years, it still highly depends on the results of MS identification, which has its inherent drawbacks, including over representation of highly expressed proteins/peptides and incomplete sampling. Moreover, the sensitivity and throughput of mass spectrometers are also important factors to maximize the benefits of proteogenomic approaches.

Shigella flexneri is the primary causative agent of endemic shigellosis in developing countries [24]. Its genome shares a large proportion of chromosomal genes with the model organism E. coli. Since 2002, the genomes of four representative strains of species in the family Shigella spp. have been sequenced $[25,26]$. As such, it is an attractive target for proteogenomic annotation. In this study, we applied high-throughput shotgun proteomic technology to explore the comprehensive protein expression profile of $S$. flexneri 2a str.301. We completely validated 823 protein products, including hundreds of hypothetical proteins. We also corrected several start sites with the help of our original $\mathrm{N}$-terminal extension database. Furthermore, certain novel open reading frames (ORFs) were confirmed by combining MS analysis and RT-PCR. Our findings suggest that current genome annotations are not yet complete, and that proteogenomic tools have the potential to validate and complement genome wide annotation.

\section{Results and Discussion}

Validation of annotated ORFs in the S. flexneri 2a str.301 genome

Raw MS/MS data were used to search a database containing all six possible reading frames of the entire $S$. flexneri 2a str.301 genome, using Mascot version 2.2. Applying the filtering criteria described in the Methods section, 823 ORFs from all experiments were unambiguously assigned, of which 811 were previously annotated in the S. flexneri 2a str.301 genome database from NCBI. (Additional file 1, Table S1). On average, between 2 or 3 peptides were used to identify each ORF, and the amino acid sequence coverage for the detected ORFs averaged 13\%. The distribution patterns of $\mathrm{pI}, \mathrm{Mr}$, and grand average of hydropathicity (GRAVY) of the identified proteins were similar to those of all S. flexneri 2a str.301 annotated proteins (Additional file 2, Figure S1-A, B, C). For example, the pI patterns of the identified proteins had the characteristic bimodal distribution, which was previously observed for bacterial and archaeal genomes [27]. Moreover, these proteins (20 of 22 groups in clusters of orthologous groups of proteins, COGs) were involved in nearly all major biological processes (Additional file 2, Figure S2-A, B). Hypothetical proteins were likely to have been annotated incorrectly because of the lack of experimental evidence, and required further experimental validation. In our study, 187 hypothetical or putative ORFs were validated at the protein level, representing $10 \%$ of the 1944 predicted hypothetical proteins of S. flexneri 2a str.301. This was below the average detection rate of all other annotated proteins. Thus, these data suggested that a certain proportion of the hypothetical protein products do not exist in the organism, and represent misannotation of the corresponding genomic region $[7,17]$. The rest of the peptides that were detected with MS but did not match any annotated protein, are analyzed in detail below. A complete list of identified peptides and their quality scores are given in Additional file 1, Table S1.

\section{Correction of gene annotation errors Correction of start codon errors}

Traditionally, it has been difficult to correctly identify the TSS within a given sequence. For example, a previous study of 143 annotated prokaryotic genomes showed that approximately $60 \%$ of the genes might have incorrectlyassigned TSSs [2]. While accurate prediction of TSSs is critical for defining protein sequences, as well as intergenic regions that might contain transcriptional regulatory elements [16]. TSSs were usually verified by N-terminal sequencing analysis. This method was often technically demanding and was not amenable to the majority of proteins with 'blocked', and therefore inaccessible, N-termini [28]. To amend the approximate location of TSSs in these 
sequences, we developed a proteomic strategy that is simpler than $\mathrm{N}$-terminal sequencing and is also capable of high-throughput analysis, as it is possible that wrongly assigned start sites could be validated and corrected in a single experiment using this method.

All MS-derived peptides were screened against both the S. flexneri 2a str.301 protein database (downloaded from $\mathrm{NCBI}$ ) and the customized N-terminal extension database (see Methods section). Peptide hits using the latter indicated that the 5 ' end of the corresponding gene should be expanded. As a result, three genes ( $y h d p, y e b j$, and $s m p A$ ) were identified as having true start codons upstream of their current start codons (Table 1; Additional file 2, Figure S3). In addition, by performing a BLASTP search against GenBank, the N-terminus extended proteins other than the original proteins shared higher similarities with their homologs in other bacteria (data not shown). Moreover, we successfully designed primers based on the Nterminal extension region for RT-PCR experiments to confirm the existence of the three extended genes (Additional file 2, Figure S4), suggesting that the N-terminal extensions inferred by our method were reliable. The initial codons of all three genes were corrected and updated in GenBank entries based on our new evidence. This original strategy of combining both $\mathrm{N}$-terminal proteomic analysis and transcriptional verification represents an effective and promising means for experimental identification of TSSs. We expect that this strategy can be applied to other organisms.

\section{Correction of sequencing errors}

Although genome sequencing technologies have made great progress in the last 10 years, none of these nextgeneration sequencing methods are $100 \%$ accurate. There are usually a few wrong bases in an otherwise accurate genome. With the aid of proteogenomic tools, we could uncover genes that contained certain avoidable sequencing errors, which usually led to erroneous annotations. For example, we found an ORF (fus $A$ ) in S. flexneri $2 a$ str.301, which was 240 bp shorter at the 3 ' end than its homologs in other Shigella genomes. However, our MS/ MS data identified peptides matching the missing part of fusA (BIO01150) in S. flexneri 2a str.301 (Figure 1A). To test if a stop codon mutation resulted from a sequencing error, we re-sequenced the coding region of fus $A$ and found that the guanine at genome position 3, 440, 920 was previously recognized as thymine, because of a mistake in the initial genome sequencing project. This sequencing error led to a transformation from GAA (coding Glu) to the premature termination codon TAA (Figure 1A). As a result, the 3' end of the fusA gene annotated in S. flexneri 2a str.301 should be extended from 3, 440,918 to $3,440,678$. Importantly, this gene is now seen to encode a full-length protein product.
Bacterial pseudogenes were originally considered to be infrequent. Despite having DNA sequences similar to those of known genes, pseudogenes were regarded as disabled copies of functional genes [29]. Nonetheless, we detected two peptide segments matching the protein product of the $z w f$ (BIO80170) pseudogene, which was orthologous to $E$. coli K12 glucose-6-phosphate dehydrogenase (Figure 1B). Was the $z w f$ gene in S. flexneri 2a str.301 a true pseudogene? Further re-sequencing of the regional genomic sequence revealed that there was an extra adenine insertion into the coding region of $z w f$ in the original S. flexneri 2a str.301 genome sequence, which resulted in frame-shift introducing a premature stop codon (Figure 1B). As such, based on our proteogenomic finds, the $z w f$ pseudogene in S. flexneri 2a str.301 was revised to encode a functional full-length product. The yraJ gene, which encodes an outer membrane usher protein in other enterobacteria, was disrupted by an IS2 insertion sequence in S. flexneri 2a str.301. Using the six-reading-frame database search, we identified this pseudogene's premature protein product (BIO11778). Its transcript was also detected by RT-PCR (Additional file 2 , Figure S5). Previous studies revealed that the intact usher protein assembled in the $\mathrm{OM}$ as a dimeric secretion complex [30]. From an evolutionary standpoint, it has been considered that transcribed/translated pseudogenes were not necessarily without function. How the premature protein functions remains to be determined.

\section{Discovery of novel ORFs}

The most striking result of our study was the identification of novel ORFs. All assigned ORFs were aligned with the current annotated ORFs of S. flexneri 2a str.301 using BLASTP, and those that aligned with annotated proteins were discarded. As a result, we detected 7 novel ORFs that were not predicted in S. flexneri 2a str.301 by any other annotation pipelines (see Table 2). Among these novel ORFs, four ORFs have orthologs in other closelyrelated organisms, which allowed substantial crossspecies validation of the new genes. Significantly, the other three ORFs were completely novel genes that had no homology with other annotated proteins from any species.

We focused on the seven novel genes to further investigate why they escaped computational prediction. First, these novel ORFs were relatively short. To our knowledge, short CDSs (especially less than 150 nucleotides) are among the most difficult genomic features to predict and are often missed during the annotation process due to conservative calls [8]. On the other hand, most of the identified novel ORFs were partially or entirely overlapped by annotated longer ORFs (Table 2; Additional file 2, Figure S6). For gene prediction software, the 
Table $1 \mathrm{~N}$-terminal extension of three genes

\begin{tabular}{cccccccc}
\hline Gene & Tag & Predicted start site & Updated state site & Old start codon & New start codon & Peptides matching N-terminal extension database & Peptide score \\
\hline yhdP & BlO47422 & 3382990 & 3383830 & GTG & GTG & DLTFWQLR \\
yebJ & Bl000465 & 1434566 & 1433987 & GTG & ATG & IGIFQDLVDR & 55 \\
& & & & & & VDLDGNPCGELDEQHVEHAR & 101 \\
smpA & Blo00925 & 2752334 & 2752145 & ATG & ATG & WYRPDINQGNYLTANDVSK & 85 \\
\hline
\end{tabular}




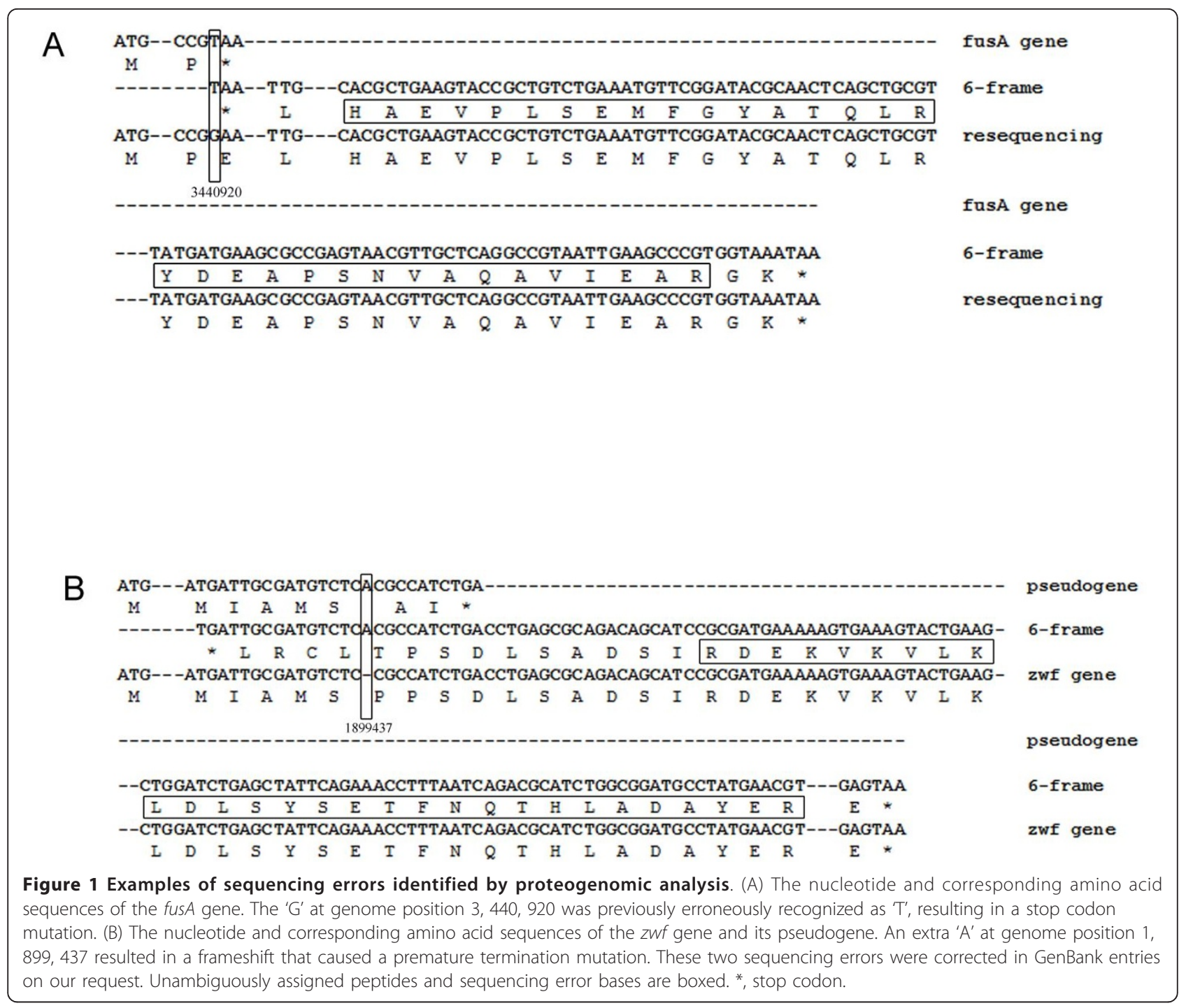

percentage of missing genes is strongly correlated with the frequency of gene overlaps. In Glimmer, the maximum overlap length is set to 30 bp by default [31]. Generally, the relatively longer ORF rather than its overlapping genes is likely to be retained. Unfortunately, those omitted overlapping genes might be true genes [23]. As Figure S6 shown, generally there were four patterns for the relative location of overlapping gene pairs.

Table 2 Characteristics of seven novel ORFs

\begin{tabular}{|c|c|c|c|c|}
\hline Gene tag & Strand & $\begin{array}{c}\text { Length } \\
\text { (AA) }\end{array}$ & Overlaps $^{\mathrm{a})}$ & Annotation in other enterobacteria \\
\hline $\mathrm{BIO01608^{ \textrm {b } ) }}$ & + & 80 & No & Hypothetical protein \\
\hline $\mathrm{BIO50043^{ \textrm {b } ) }}$ & - & 365 & Partial (S) & Sulfate/thiosulfate transporter subunit \\
\hline $\mathrm{BIO} 07235^{\mathrm{b})}$ & + & 25 & Partial (S) & None \\
\hline $\mathrm{BlO} 43803^{\mathrm{b})}$ & - & 496 & Partial (C) & Hypothetical protein \\
\hline $\mathrm{BIO68373}$ & - & 59 & Nested (C) & Conserved hypothetical protein \\
\hline BIO58539 & - & 86 & Nested (S) & None \\
\hline $\mathrm{BlO} 48527$ & - & 36 & Nested (S) & None \\
\hline
\end{tabular}

a) No, ORFs not overlapping other genes; Partial (C), ORFs partially overlapping known genes on the complementary strand; Partial (S), ORFs partially overlapping known genes on the same strand; Nested (C), ORFs completely contained within known genes on the complementary strand; Nested (S), ORFs completely contained within known genes on the same strand, but in a different frame.

b) The transcripts of novel ORFs were confirmed by RT-PCR assay. 
Of the seven novel ORFs, one ORF (BIO01608) had no overlap with known genes and other three ORFs (BIO50043, BIO07235, BIO43803) respectively belonged to pattern I or pattern II, whose transcripts were easy to be verified by RT-PCR assays. The results showed that the transcripts of four ORFs were specifically detected (Figure 2), and additional sequencing of these PCR products confirmed their identity. The rest three ORFs (BIO58539, BIO48527, BIO68373) were entirely contained within the coding region of certain longer known genes (Pattern III or Pattern IV), referred to as "nested" genes. Although nested genes are quite rare in prokaryotic genomes, this kind of gene arrangement is beginning to be recognized, such as setBA / pic in S. flexneri 2a [32,33], ins $5 B$ /ins $5 A$ and $h g t A / y a a W$ in $E$. coli [34,35], and Pfl01_0939/cosA in P. Fluorescens [36]. The existence of nested genes increases the organizational complexity of the genome structure, so it is not practical to investigate all gene arrangements during conventional genome annotation. As such, proteogenomic methods offer a promising avenue toward the experimental validation of nested genes at the protein level [37].

Short CDSs remain largely unknown, even though small peptides encoded by short genes are involved in diverse functions, such as secretion, stress responses, metabolism, and gene regulation in bacteria [38,39]. We also examined the gene structure of each of the seven novel genes. In our study, there were no identifiable

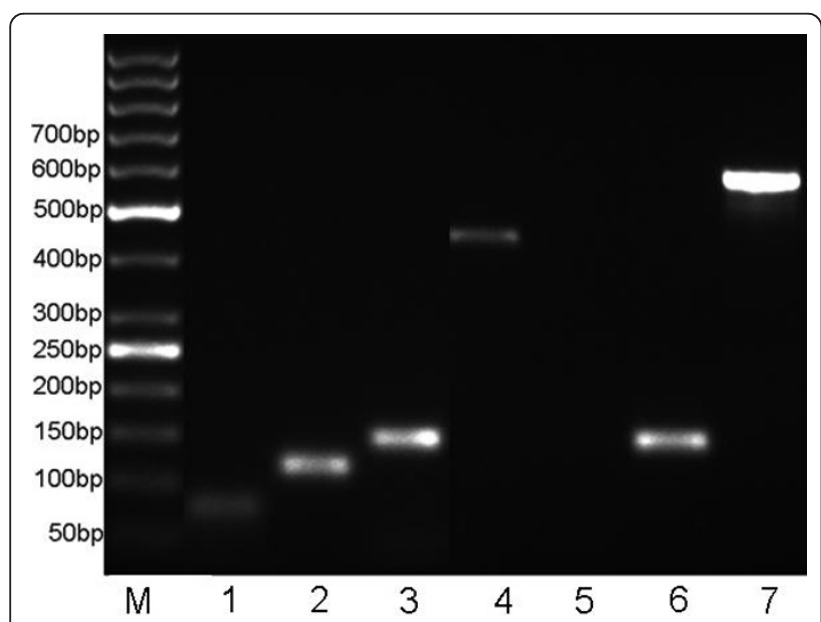

Figure 2 Validating MS data using RT-PCR. RNA fragments of the expected sizes were observed, indicating that these un-annotated genes are transcribed. Figure shows the RT-PCR verification results for six novel genes. Amplified PCR products were electrophoresed on a $2.5 \%$ agarose gel and visualized by ethidium bromide staining.

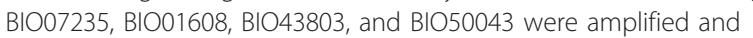
loaded in lanes 1-4, respectively; a negative control (noncoding DNA sequence) was loaded in lane 5 (cDNA as template) and lane 6 (genomic DNA as template); Lane 7, positive control

(housekeeping gene, ipaD); Lane M, GeneRuler'TM 50 bp DNA Ladder (Fermentas $\mathrm{GmbH}$, Germany). functional domains in the seven novel ORFs, with the exception of BIO01608 and BIO50043. BIO01608 contains an YmgB superfamily conserved domain, which is involved in biofilm development and stability. BIO50043 contains an ABC_CysA_sulfate_importer conserved domain, which is involved in sulfate import, and whose ortholog in E. coli is annotated as a sulfate/thiosulfate transporter subunit. Overlapping gene pairs were conserved among organisms for specific genes and functions. In addition, it was generally assumed that their expressions were correlated with host genes, which would reduce the need for more complex regulatory pathways and thus the regulation of gene expression would be more effective $[1,40]$. For example, of the setBA / pic nested gene pair in S. flexneri $2 \mathrm{a}$, the pic gene encodes mucinase, which is involved in mucosal colonization, and set $1 A$ and set $1 B$ encode the two subunits of the ShET1 enterotoxin. The two partners were likely to be expressed reciprocally and function cooperatively [41], which aided our further investigation of the function of nested gene pair. Exploring these novel nested genes' biological functions and their coordination with host genes are under investigation.>

\section{Conclusions}

In our study, the detection of annotation errors, such as incorrect start sites assignment, sequencing errors, and wrongly annotated pseudogenes, would prevent misannotation from being multiplied in future versions of the $S$. flexneri 2a str.301 genome. The findings of novel ORFs would also provide a new clue to conduct functional research. Moreover, some of the novel ORFs were identified as overlapping genes, which increases our understanding of the complexity of the genome structure and reveals the underestimation of such gene arrangements. This updated dataset would be very helpful for those interested in this pathogen to unearth certain information previously omitted. With the rapid development of proteomic technology, all sequence-based genome projects could be supplemented by the proteogenomic analysis.

\section{Methods}

\section{Strain and culture conditions}

Frozen S. flexneri 2a, str.301 (kindly provided by the ICDC, China CDC) cell stocks were streaked onto tryptic soy agar containing $0.01 \%$ Congo red. An individual red colony was subsequently transferred into tryptic soy broth (TSB) and grown overnight at $37^{\circ} \mathrm{C}$ with rotary shaking at $200 \mathrm{rpm}$. The overnight culture was diluted 1:50 in fresh TSB and incubated under the same conditions until $\mathrm{OD}_{600}=0.6-1.0$. Cells were harvested by 8 min centrifugation at $2,500 \times g$ at $4^{\circ} \mathrm{C}$ and then washed twice ice-cold $50 \mathrm{mM}$ Tris- $\mathrm{HCl}, \mathrm{pH}$ 7.3. The pelleted cells were frozen at $-20^{\circ} \mathrm{C}$ until required. 


\section{Sample pre-fractionation procedures}

Bacterial cells were resuspended in $100 \mathrm{mM}$ Tris- $\mathrm{HCl}$ buffer ( $\mathrm{pH}$ 8.5), containing $7 \mathrm{M}$ Urea, $2 \mathrm{M}$ Thiourea, a protease inhibitor cocktail tablet (Roche Diagnostics, Germany), and Benzonase Nuclease (25 U/ml, Sigma, USA), and then ruptured by ultrasonication. The unbroken cells were removed by centrifugation at 4,000 $\times g$ for $10 \mathrm{~min}$ at $4^{\circ} \mathrm{C}$. The supernatant was diluted with ice cold $100 \mathrm{mM} \mathrm{Na}_{2} \mathrm{CO}_{3}$ (pH 11.5) to a final pH 11 and stirred slowly on ice for $1 \mathrm{~h}$. The supernatant was further collected by ultracentrifugation in a Beckman SW 40Ti rotor at an average of $150,000 \times \mathrm{g}$ for $1 \mathrm{~h}$ at $4^{\circ} \mathrm{C}$. The supernatant was analyzed for cytosolic protein components. The membrane pellet was resuspended and washed twice in ice-cold $100 \mathrm{mM} \mathrm{Na}_{2} \mathrm{CO}_{3}(\mathrm{pH}$ 11.5) at $4^{\circ} \mathrm{C}$. Finally, the washed membrane sheets were pelleted by ultracentrifugation at an average of $150,000 \times g$ for $45 \mathrm{~min}$ and resuspended in $100 \mathrm{mM} \mathrm{NH}_{4} \mathrm{HCO}_{3}$ containing $7 \mathrm{M}$ Urea and $2 \mathrm{M}$ Thiourea [42]. Cytosolic and membrane fractions were measured for protein content using a bicinchoninic acid (BCA) assay. Both of fraction samples were analyzed in parallel and replicated three times.

\section{In-solution digest}

Cytosolic and membrane fractions were reduced in the presence of $10 \mathrm{mM} \mathrm{DTT}$ at $37^{\circ} \mathrm{C}$ for $45 \mathrm{~min}$, and then alkylated in the presence of $50 \mathrm{mM}$ iodacetamide at room temperature in the dark for $30 \mathrm{~min}$. The reaction products were diluted to $1 \mathrm{M}$ urea and digested with trypsin (1:50 w/w, modified sequencing grade, Promega, USA) overnight at $37^{\circ} \mathrm{C}$. Peptides were desalted using an Oasis HLB extraction cartridge (Waters, USA). All peptide fractions were concentrated with a Speed-vac centrifuge (Eppendorf, Germany) and resolubilized in $0.1 \%$ TFA for the following two-dimensional liquid chromatography matrix-assisted laser desorption/ionization (2D LC-MALDI) analysis.

\section{D LC-MALDI analysis}

Digested peptide mixtures were separated using the Ultimate 3000 HPLC system (Dionex-LC-Packings, USA) coupled with a PROTEINEER fc LC-MALDI fraction collector (Bruker, Germany). The HPLC system consisted of a strong cation exchange (SCX) column $(300 \mu \mathrm{m}$ id POROS 10S Column, Dionex) and a C18 reverse-phase microcapillary column (PepMap100 C18, $300 \mu \mathrm{m}, 100 \AA$, Dionex). The flow rate through the column was $2 \mu \mathrm{l} / \mathrm{min}$. The solutions used were as follows: $0.05 \%$ TFA in water (buffer A), 0.04\% TFA in 80\% ACN (buffer B). A sample of the desired peptides digest was loaded onto the SCX column. $\mathrm{NaCl}$ of different concentration at $0.5,1,2,3,5$, $10,25,50,100,200,500,1000 \mathrm{mM}$ was used to displace peptide fractions from the SCX column onto the RP column, respectively. Each case was synchronized with a 90 min RP gradient. Gradient conditions: isocratic prerun at 4\% B, 0-5 min; linear gradient 4-65\% B, 5-65 min; $65-100 \% \mathrm{~B}, 65-70 \mathrm{~min}$, column wash at $100 \% \mathrm{~B}, 70-75$ min; re-equilibrate the column at 4\% B, 75-90 min. Online MALDI spotting of LC fractions was carried as follows: number of fractions, 384 (covering the gradient 4-65\% B); MALDI target, Pre-spotted disposable AnchorChip PAC 384 HCCA target (Bruker, Germany); fraction width, $15 \mathrm{~s}(500 \mathrm{nl})$. MS spectra were automatically acquired on an Ultraflex III MALDI-TOF/TOF mass spectrometer (Bruker Daltonics, Germany) in the positive reflection mode under the control of Compass 1.2 and WARP-LC 1.0 software (Bruker Daltonics, Germany). The parameter settings were: $20 \mathrm{kV}$ accelerating voltage and $23 \mathrm{kV}$ reflecting voltage; MS and MS/MS mass range: $m / z 700-4000$ and 50-2000, respectively; Detected peptide compounds with a signal-to-noise ratio higher than 10 were subjected to MALDI-time of flight (TOF) MS/MS analysis.

\section{In-house database construction}

We translated the S. flexneri 2a str.301 genome (downloaded from NCBI) into all six possible reading frames, generating a set of all possible peptides (larger than 15 amino acids) that could be encoded. Sequences for common contaminants from two collections (248 from Max Planck Institute of Biochemistry, 112 from the Global Proteome Machine Organization Common Repository of Adventitious Protein), were merged into one (total 338 unique entries) and appended to the end of the above target database FASTA file. The final database had 90330 entries. To detect potential extended TSSs of the predicted coding sequences, we constructed a specialized Nterminal extension database, using a similar strategy as previously described [16] with some changes. The database took into account all currently annotated CDSs from the $S$. flexneri $2 a$ str.301 genome. The region upstream of each CDS was scanned until an in-frame stop codon was identified. Then, the in-frame codons downstream of this stop codon were scanned for the first location of a start codon (ATG, GTG or TTG). The peptide from the new start codon to the 33rd amino acid residue downstream of original start site was collected into the extension database, except for those CDSs whose start codon was the same as the previous annotation. As a result, 1311 peptides were collected in the customized extension database (Additional file 3, Table S2).

\section{Data evaluation}

MS/MS data were searched using Biotools 3.1 software (Bruker Daltonics, Germany) with MASCOT 2.2 plugin http://www.matrixscience.com against the six reading frame translation of $S$. flexneri 2a str.301 genome. All 
MS/MS spectra were deposited into the PRIDE database [43]http://www.ebi.ac.uk/pride/ and could be downloaded from this URL: http://www.mgc.ac.cn/Resources/ mzXML_S.flexneri_WARP-LC.tgz. The following search parameters were applied: max missed cleavage: 1 ; fixed modification: Carbamidomethylation $(\mathrm{C})$; variable modification: Oxidation (M), Carbamyl (N-term), Deamidated (NQ); precursor ion mass tolerance: $\pm 50 \mathrm{ppm}$; fragment mass tolerance: $\pm 0.6 \mathrm{Da}$. Decoy searches were performed using the automated 'Decoy' search option from Mascot. In this strategy, Mascot will generate and search a random version of each target database protein. The false discovery rate (FDR) is calculated as follows:

$$
\text { FDR = Decoy hits (FP)/Target hits (FP + TP) . }
$$

We tweaked the peptide significance threshold (at most 0.01 ) to control the FDR value under $1 \%$. Under these criteria, all the proteins with at least one unique peptide identification at $p<0.01$ were considered likely to be present in the sample. Additionally, total proteins identified by a single peptide and all novel protein identifications could not be accepted unless their corresponding MS/MS spectra passed the manual validation. All spectra used for annotated ORF identifications based on unique peptides (ion score < 45), as well as all those of novel ORFs are shown in Additional file 4.

\section{RT-PCR}

Total RNA of S. flexneri 2a str.301 was extracted using the SV Total RNA Isolation System Kit (Promega, USA) following the manufacturer's protocol. Total RNA was treated with RQ1 RNase-free DNase (Promega, USA) to remove residual genomic DNA, followed by heat inactivation of the endonuclease. cDNA synthesis was performed from $1 \mu \mathrm{g}$ of RNA using the SuperScript ${ }^{\mathrm{TM}}$ III Reverse Transcriptase (Invitrogen, USA) according to the manufacturer's protocol. PCR was performed using $1 \mu \mathrm{l}$ of the reverse transcription reaction as a starting material according to standard procedures. PCR cycling parameters were typically $4 \mathrm{~min}$ at $94^{\circ} \mathrm{C} ; 30$ cycles of $30 \mathrm{~s}$ at $94^{\circ} \mathrm{C}, 30 \mathrm{~s}$ at $55^{\circ} \mathrm{C}, 30 \mathrm{~s}$ at $72^{\circ} \mathrm{C}$; and a final $10 \mathrm{~min}$ extension at $72^{\circ} \mathrm{C}$. The RT-PCR assay was run with the housekeeping gene (ipaD) as a positive control and a noncoding DNA sequence (from 417, 540 to 417, 690 in the S. flexneri 2a str.301 genome) as the negative control. Gene-specific primers used to amplify the target genes are listed in Additional file 5, Table S3.

\section{Additional material}

Additional file 1: Supplementary Table S1, All proteins identified by MS analysis. This file contains detailed information of all identified proteins in our study.
Additional file 2: Supplementary Figures. This file contains supplementary Figures S1-6. Figure S1 illustrates the patterns of pl, Mr, and GRAVY value of identified/annotated proteins. Figure S2 illustrates COGs functional categories of identified/annotated proteins. Figure S3 shows the information about $\mathrm{N}$-terminal extension of three genes. Figure S4 shows RT-PCR results of three extended genes. Figure S5 shows RT-PCR results for BIO11778. Figure S6 illustrates patterns for relative location of novel ORFs overlapping known genes.

Additional file 3: Supplementary Table S2, List of candidate $\mathrm{N}$-terminal extension genes. This file contains a list of genes that are likely to be extended at the $\mathrm{N}$-terminus in the S. flexneri 2a str.301 genome. Each entry's information includes locus tag, extension region: genome position of region from $\mathrm{N}$-terminal new start codon to the 33rd amino acid residue downstream of original start site for each extended gene, and the peptide sequence corresponding to extension region.

Additional file 4: Manually validated MS/MS spectra. This file shows all MS/MS spectra of peptides matching to annotated proteins that had a single peptide hit (ion score < 45) and un-annotated novel proteins.

Additional file 5: Supplementary Table S3. Table S3 shows a list of primers used in this article.

\section{Acknowledgements}

Authors thank Dr. Jian Yang and Dr. Tao Liu (Institute of Pathogen Biology) for their kind technical assistances. This work was supported in part by the major project 'AIDS, viral hepatitis and other major infectious diseases prevention and control' (Grant No.2009ZX10004-102) from the Ministry of Health of China, the National Basic Research Program (Grant

No.2011CB504901) from the Ministry of Science and Technology of China, the National Natural Science Fund (Grant No. 30700036) from the National Natural Science Foundation of China, and an intramural grant from the Institute of Pathogen Biology, Chinese Academy of Medical Sciences (2008IPB011).

\section{Author details}

'State Key Laboratory for Molecular Virology and Genetic Engineering, Institute of Pathogen Biology, Chinese Academy of Medical Sciences \& Peking Union Medical College, Beijing, PR China. ${ }^{2}$ Department of Biological Engineering, College of Life Sciences, Hebei United University, Tangshan City, Hebei Province, P.R. China.

\section{Authors' contributions}

LZ carried out the proteomic experiment, participated in the sequence alignment, and drafted the manuscript. LL carried out the RT-PCR assay. WL participated in the MS analysis. CW participated in the design of the study, performed the statistical analysis, and helped to draft the manuscript. QJ conceived the study, and participated in its design and coordination. All authors read and approved the final manuscript.

\section{Competing interests}

The authors declare that they have no competing interests.

Received: 6 May 2011 Accepted: 28 October 2011

Published: 28 October 2011

\section{References}

1. Johnson ZI, Chisholm SW: Properties of overlapping genes are conserved across microbial genomes. Genome Res 2004, 14(11):2268-2272.

2. Nielsen $P$, Krogh A: Large-scale prokaryotic gene prediction and comparison to genome annotation. Bioinformatics 2005, 21(24):4322-4329.

3. Reeves GA, Talavera D, Thornton JM: Genome and proteome annotation: organization, interpretation and integration. J $R$ Soc Interface 2009, 6(31):129-147.

4. Gallien S, Perrodou E, Carapito C, Deshayes C, Reyrat JM, Van Dorsselaer A, Poch O, Schaeffer C, Lecompte O: Ortho-proteogenomics: multiple proteomes investigation through orthology and a new MS-based protocol. Genome Res 2009, 19(1):128-135. 
5. Aivaliotis M, Gevaert K, Falb M, Tebbe A, Konstantinidis K, Bisle B, Klein C, Martens L, Staes A, Timmerman E, et al: Large-scale identification of Nterminal peptides in the halophilic archaea Halobacterium salinarum and Natronomonas pharaonis. J Proteome Res 2007, 6(6):2195-2204

6. Palleja A, Harrington ED, Bork P: Large gene overlaps in prokaryotic genomes: result of functional constraints or mispredictions? BMC Genomics 2008, 9:335.

7. Jaffe JD, Berg HC, Church GM: Proteogenomic mapping as a complementary method to perform genome annotation. Proteomics 2004, 4(1):59-77.

8. Ansong C, Purvine SO, Adkins JN, Lipton MS, Smith RD: Proteogenomics: needs and roles to be filled by proteomics in genome annotation. Brief Funct Genomic Proteomic 2008, 7(1):50-62.

9. Renuse S, Chaerkady R, Pandey A: Proteogenomics. Proteomics 2011, 11(4):620-630.

10. de Souza GA, Softeland T, Koehler CJ, Thiede B, Wiker HG: Validating divergent ORF annotation of the Mycobacterium leprae genome through a full translation data set and peptide identification by tandem mass spectrometry. Proteomics 2009, 9(12):3233-3243.

11. Gupta N, Tanner S, Jaitly N, Adkins JN, Lipton M, Edwards R, Romine M, Osterman A, Bafna V, Smith RD, et al: Whole proteome analysis of posttranslational modifications: applications of mass-spectrometry for proteogenomic annotation. Genome Res 2007, 17(9):1362-1377.

12. Jaffe JD, Stange-Thomann N, Smith C, DeCaprio D, Fisher S, Butler J, Calvo S, Elkins T, FitzGerald MG, Hafez N, et al: The complete genome and proteome of Mycoplasma mobile. Genome Res 2004, 14(8):1447-1461.

13. Xia D, Sanderson SJ, Jones AR, Prieto JH, Yates JR, Bromley E, Tomley FM, Lal K, Sinden RE, Brunk BP, et al: The proteome of Toxoplasma gondii: integration with the genome provides novel insights into gene expression and annotation. Genome Biol 2008, 9(7):R116.

14. Baerenfaller K, Grossmann J, Grobei MA, Hull R, Hirsch-Hoffmann M, Yalovsky S, Zimmermann P, Grossniklaus U, Gruissem W, Baginsky S: Genome-scale proteomics reveals Arabidopsis thaliana gene models and proteome dynamics. Science 2008, 320(5878):938-941.

15. Fermin D, Allen BB, Blackwell TW, Menon R, Adamski M, Xu Y, Ulintz $P$, Omenn GS, States DJ: Novel gene and gene model detection using a whole genome open reading frame analysis in proteomics. Genome Biol 2006, 7(4):R35.

16. Rison SC, Mattow J, Jungblut PR, Stoker NG: Experimental determination of translational starts using peptide mass mapping and tandem mass spectrometry within the proteome of Mycobacterium tuberculosis. Microbiology 2007, 153(Pt 2):521-528.

17. Ishino $Y$, Okada H, Ikeuchi M, Taniguchi H: Mass spectrometry-based prokaryote gene annotation. Proteomics 2007, 7(22):4053-4065.

18. Kalume DE, Peri S, Reddy R, Zhong J, Okulate M, Kumar N, Pandey A: Genome annotation of Anopheles gambiae using mass spectrometryderived data. BMC Genomics 2005, 6:128.

19. Lamontagne J, Beland M, Forest A, Cote-Martin A, Nassif N, Tomaki F, Moriyon I, Moreno E, Paramithiotis E: Proteomics-based confirmation of protein expression and correction of annotation errors in the Brucella abortus genome. BMC Genomics 2010, 11:300

20. de Groot A, Dulermo R, Ortet P, Blanchard L, Guerin P, Fernandez B, Vacherie $B$, Dossat $C$, Jolivet $E$, Siguier $P$, et al: Alliance of proteomics and genomics to unravel the specificities of Sahara bacterium Deinococcus deserti. PLoS Genet 2009, 5(3):e1000434.

21. Wei C, Peng J, Xiong Z, Yang J, Wang J, Jin Q: Subproteomic tools to increase genome annotation complexity. Proteomics 2008, 8(20):4209-4213.

22. Findlay GD, MacCoss MJ, Swanson WJ: Proteomic discovery of previously unannotated, rapidly evolving seminal fluid genes in Drosophila. Genome Res 2009, 19(5):886-896.

23. Payne SH, Huang ST, Pieper R: A proteogenomic update to Yersinia: enhancing genome annotation. BMC Genomics 2010, 11:460.

24. Kotloff KL, Winickoff JP, Ivanoff B, Clemens JD, Swerdlow DL, Sansonetti PJ, Adak GK, Levine MM: Global burden of Shigella infections: implications for vaccine development and implementation of control strategies. Bull World Health Organ 1999, 77(8):651-666.

25. Jin Q, Yuan Z, Xu J, Wang Y, Shen Y, Lu W, Wang J, Liu H, Yang J, Yang F, et al: Genome sequence of Shigella flexneri 2a: insights into pathogenicity through comparison with genomes of Escherichia coli K12 and 0157. Nucleic Acids Res 2002, 30(20):4432-4441.
26. Yang F, Yang J, Zhang $X$, Chen $L$, Jiang $Y$, Yan $Y$, Tang $X$, Wang J, Xiong $Z$, Dong J, et al: Genome dynamics and diversity of Shigella species, the etiologic agents of bacillary dysentery. Nucleic Acids Res 2005, 33(19):6445-6458.

27. VanBogelen RA, Schiller EE, Thomas JD, Neidhardt FC: Diagnosis of cellular states of microbial organisms using proteomics. Electrophoresis 1999, 20(11):2149-2159.

28. Link AJ, Robison K, Church GM: Comparing the predicted and observed properties of proteins encoded in the genome of Escherichia coli K-12. Electrophoresis 1997, 18(8):1259-1313.

29. Lerat $\mathrm{E}$, Ochman $\mathrm{H}$ : Recognizing the pseudogenes in bacterial genomes. Nucleic Acids Res 2005, 33(10):3125-3132.

30. Li H, Qian L, Chen Z, Thibault D, Liu G, Liu T, Thanassi DG: The outer membrane usher forms a twin-pore secretion complex. J Mol Biol 2004, 344(5):1397-1407.

31. Delcher AL, Harmon D, Kasif S, White O, Salzberg SL: Improved microbial gene identification with GLIMMER. Nucleic Acids Res 1999, 27(23):4636-4641.

32. Al-Hasani K, Rajakumar K, Bulach D, Robins-Browne R, Adler B, Sakellaris H: Genetic organization of the she pathogenicity island in Shigella flexneri 2a. Microb Pathog 2001, 30(1):1-8.

33. Fasano A, Noriega FR, Maneval DR Jr, Chanasongcram S, Russell R, Guandalini S, Levine MM: Shigella enterotoxin 1: an enterotoxin of Shigella flexneri $2 a$ active in rabbit small intestine in vivo and in vitro. $J$ Clin Invest 1995, 95(6):2853-2861.

34. Sawers RG: Transcript analysis of Escherichia coli K-12 insertion element IS5. FEMS Microbiol Lett 2005, 244(2):397-401.

35. Delaye $L$, Deluna A, Lazcano A, Becerra A: The origin of a novel gene through overprinting in Escherichia coli. BMC Evol Biol 2008, 8:31.

36. Silby MW, Levy SB: Overlapping protein-encoding genes in Pseudomonas fluorescens Pf0-1. PLoS Genet 2008, 4(6):e1000094.

37. Kim W, Silby MW, Purvine SO, Nicoll JS, Hixson KK, Monroe M, Nicora CD, Lipton MS, Levy SB: Proteomic detection of non-annotated proteincoding genes in Pseudomonas fluorescens Pf0-1. PLoS One 2009, 4(12): e8455.

38. Alix E, Blanc-Potard AB: Hydrophobic peptides: novel regulators within bacterial membrane. Mol Microbiol 2009, 72(1):5-11.

39. Ibrahim M, Nicolas $P$, Bessieres $P$, Bolotin A, Monnet V, Gardan R: A genome-wide survey of short coding sequences in streptococci. Microbiology 2007, 153(Pt 11):3631-3644.

40. Kumar A: An overview of nested genes in eukaryotic genomes. Eukaryot Cell 2009, 8(9):1321-1329.

41. Behrens M, Sheikh J, Nataro JP: Regulation of the overlapping pic/set locus in Shigella flexneri and enteroaggregative Escherichia coli. Infect Immun 2002, 70(6):2915-2925.

42. Wei C, Yang J, Zhu J, Zhang X, Leng W, Wang J, Xue Y, Sun L, Li W, Jin Q: Comprehensive proteomic analysis of Shigella flexneri 2a membrane proteins. J Proteome Res 2006, 5(8):1860-1865

43. Vizcaino JA, Cote R, Reisinger F, Foster JM, Mueller M, Rameseder J, Hermjakob $H$, Martens L: A guide to the Proteomics Identifications Database proteomics data repository. Proteomics 2009, 9(18):4276-4283.

doi:10.1186/1471-2164-12-528

Cite this article as: Zhao et al:: A proteogenomic analysis of Shigella flexneri using 2D LC-MALDI TOF/TOF. BMC Genomics 2011 12:528.

\section{Submit your next manuscript to BioMed Central and take full advantage of:}

- Convenient online submission

- Thorough peer review

- No space constraints or color figure charges

- Immediate publication on acceptance

- Inclusion in PubMed, CAS, Scopus and Google Scholar

- Research which is freely available for redistribution 